\title{
Evaluation of the effects of food intake on task engagement based on psychophysiological states
}

\author{
Nanako Kurogi ${ }^{1} \cdot$ Kanako Sakiyama $^{2} \cdot$ Shigeru Tominaga ${ }^{2} \cdot$ Yoshio Toyama $^{2} \cdot$ Kent Nagumo $^{1} \cdot$ Kosuke Oiwa $^{1}$. \\ Akio Nozawa ${ }^{1}$
}

Received: 15 April 2021 / Accepted: 11 October 2021 / Published online: 27 October 2021

(c) The Author(s) 2021

\begin{abstract}
The objective of this study was to evaluate the effects of food intake on performance and task engagement based on psychophysiological states. The experiment was conducted under two conditions: taking a break with his eyes opened between the repeated tracking tasks (Control), and taking a break with food intake (Intake). We found the rate of deterioration of the distance between target pointer and the mouse cursor, a performance index, showed significant differences between the two conditions. Significant differences were also found between the two conditions in all assessment items of subjective feelings. Considering the hemodynamic parameters and nasal skin temperature responses, it was found that the approach to the second task was different in the two conditions. Therefore, due to food intake between repeated tasks, psychological responses such as "Awareness" and "Refreshment" were maintained. Hence, it was confirmed that food intake stimulated positive task engagement in the task and maintained performance.
\end{abstract}

Keywords Act of intake $\cdot$ Hemodynamic reaction patterns $\cdot$ Mental health $\cdot$ Psychophysiological measurement $\cdot$ Repeated tasks $\cdot$ Task engagement

\section{Introduction}

In modern society, the deterioration of workers' mental health has become a growing social problem. Mental health problems are a significant cause of illness and disability among the working population [1]. The workplace is an important environment that affects mental health [2]. In Japan, the Workplace Reform Act was enacted in response under which companies are urgently required to improve their working environment [3]. In recent years, the

This work was presented in part at the 26th International Symposium on Artificial Life and Robotics (Online, January 21-23, 2021).

Nanako Kurogi

ngng.3127@gmail.com

Akio Nozawa

akio@ee.aoyama.ac.jp

1 Aoyama Gakuin University, 5-10-1 Fuchinobe, Chuo-ku, Sagamihara, Kanagawa, Japan

2 Meiji Holdings Co., Ltd., 1-29-1 Nanakuni, Hachioji, Tokyo, Japan immediate development of information and communication technologies (ICT) such as artificial intelligence (AI) and internet of things (IoT) has led to the increasing digitization of work. Digitalization is making work more efficient; in this regard, remote work is also being promoted. However, employees are forced to work for long hours in physical workplaces. To maintain their mental health, employees should work independently, willingly, and positively.

Workers' mental health is related to their work style. Work style has been studied from the viewpoint of work engagement and workaholism $[4,5]$. A "Workaholism" is defined as a person who is obsessively driven to work both excessively and hard [6]. Work engagement has been proposed as a counter concept to burnout. Burnout, as a concept, describes the tendency to become exhausted and depressed as a result of expending too much energy at work [7]. The relationship between workaholism, work engagement and recovery experiences (how to rest) such as "psychological detachment from work," "relaxation" and "Control," among others have also been studied $[8,9]$. Moreover, many studies have been conducted to improve work engagement in the workplace. For example, studies have suggested good sleep $[10,11]$ and a psychological detachment from work [12]. However, work 
engagement is a concept characterized by "persistent feelings and cognitions" toward work, and not as "temporary states." In other words, work engagement is a concept that captures a state that is both sustainable and stable, although there are aspects that fluctuate over time on a daily basis [9]. Therefore, to reduce the risk of workers falling prey to mental health problems, it is necessary to focus on task engagement in the workplace. If we can quantitatively and objectively evaluate task engagement, measures can be taken to minimize mental health risks and stress had by work in the workplace. This is also expected as being applicable to understanding the work styles of remote and office work.

There are some studies that have investigated how to spend one's breaks during work, suggesting options such as engaging in a "physical task," "having a conversation," "reading," and "eating food", along with their effects on performance [13-16]. In general, we often try to refresh ourselves by eating food while at work. However, in Miki et al.'s study, the act of "eating food" had some effect on individuals' biometric information, but did not show any improvement in regard to the index concerning the state of stress; the LF/HF value [16]. Pagani et al. proposed that LF/HF values are an index of the autonomic nervous system that can quantify the balance between sympathetic and parasympathetic activity [17, 18]. The effect had by eating behaviors such as chewing and swallowing on autonomic nervous system activity would also be expected to be superimposed to this index. Therefore, it is difficult to evaluate the effect of eating on one's task engagement by only using physiological indices; thus, it is necessary to evaluate the psychological state and one's performance as well. Previously, the time series feature of task engagement concerning repeated tasks that are expected to lead to fatigue has been evaluated in terms of physiological stress coping styles [19, 20]. The physiological stress coping style is closely related to task engagement and can be broadly classified into hemodynamic reaction patterns (active stress coping and passive stress coping) according to hemodynamic parameters [21, 22].

The objective of this study was to evaluate the effects of food intake on performance and task engagement based on psychophysiological states. In this study, and to objectively and quantitatively evaluate task engagement in repetitive tasks, we measured and evaluated physiological stress coping styles as well as arousal, the stress state as based on nasal skin temperature (NST), and the psychological state, which are thought to affect performance.

\section{Experiment}

\subsection{Experimental systems}

The experimental system consisted of a non-invasive blood continuous hemodynamometer (Finometer MIDI; Finapres
Medical Systems, the Netherlands), a piece of wireless biological measuring equipment (Polymate Mini AP108; TEAC Co., Japan), an infrared thermography device (FLIR A600Series; FLIR systems Co., Ltd, the USA), a liquid crystal display (LCD) monitor, and a table and chair for the subject. The table was placed in front of the subject. In this study, an LCD monitor was placed $0.6 \mathrm{~m}$ in front of the subject and an infrared thermography device was attached to the monitor. The experiment was conducted in an experiment room in which the temperature was set to $25.53 \pm 0.79^{\circ} \mathrm{C}$ (mean $\pm \mathrm{SD}$ ). The measurements began after the subjects had been in the experiment room for at least $20 \mathrm{~min}$ in order to allow them to acclimatize to the room temperature.

The hemodynamic parameters measured in this experiment were mean blood pressure (MBP), cardiac output (CO), and total peripheral vascular resistance (TPR). The hemodynamic parameters were measured using a non-invasive blood continuous hemodynamic meter. MBP was measured by attaching a blood pressure cuff to the second joint of the left middle finger with a hemodynamometer at $1 \mathrm{~Hz}$. $\mathrm{CO}$ and TPR were calculated with the Modelflow software (BeatScope Easy; Finapres Medical Systems, the Netherlands) that applied the Modelflow method. CO is the volume of blood that is pumped out of the heart in $1 \mathrm{~min}$. TPR is the resistance of blood flow in peripheral blood vessels, which increases with peripheral vasoconstriction and increased blood viscosity. The electroencephalograms (EEG) were measured using the Polymate equipment, with a sampling frequency of $500 \mathrm{~Hz}$. The EEG signals were recorded from a midline parietal location (Pz), as defined by the international 10-20 system. The reference electrode was placed at the left ear lobe (A1). Facial thermal images (FTIs) were measured using the infrared thermography device placed 0.6 $\mathrm{m}$ away from the subjects. FTIs were created at 1-s sampling intervals. The size of each FTI was $640 \times 480$ pixels, and the temperature resolution was less than $0.05^{\circ} \mathrm{C}$. The infrared emissivity of skin was $\varepsilon=0.98$.

Four kinds of subjective feelings, namely "Comfort", "Awareness", "Vigor" and "Refreshment" were evaluated using the visual analogue scale (VAS). A VAS is a method to subjectively evaluate a psychological state, by asking subjects to select a position on a line, with a pair of adjectives set at the ends. The VAS can measure various items simultaneously with a single item by setting adjectives, and the data can be measured on a continuous scale with the subject's subjective perception.

\subsection{Procedure and conditions}

24 healthy subjects [24 men; age, $22.5 \pm 1.3$ (mean \pm SD)] participated in this study. The subjects were fully informed about the experiment procedures and the objective of this 
study before obtaining their consent to participate. The number of experiments per subject was set to once per day.

Figure 1 shows the experimental protocol. The first half of the experiment consisted of an initial 1-min resting section (Rest1), a 15-min tracking task section (Task1), and another 1-min resting section (Rest2). After a break, the second half of the experiment commenced. The second half also consisted of a 1-min resting section (Rest3), a 15-min tracking task section (Task2), a 1-min resting section (Rest4), and a 3-min resting section (Rest5) after completing the entire procedure. In each resting section, subjects were instructed to maintain a rested state with their eyes closed. Additionally, "VAS" in the Fig. 1 indicates psychological evaluation. The tracking task tracked a target moving at about 150 $\mathrm{mm} / \mathrm{s}$ in an irregular direction on the screen by operating the mouse on the desk. This protocol was conducted twice. The experiment was conducted under two conditions: taking a break with his eyes opened between the repeated tasks (Control) and taking a break with food intake (Intake). In the Intake condition, the same type of food was across subjects. The order of experimental conditions was counterbalanced across the subjects.

\subsection{Indices for analysis}

The distance difference between the target pointer and the mouse cursor (TErr) was measured as the performance index. The total amount of error was calculated as the sum of TErr for 15 min tracking task section. For this study, the rate of deterioration of TErr was calculated by dividing the total amount of error in Task 2 by the total amount of error in Task1.

The hemodynamic parameters were measured as an index of cardiovascular system activity. Task engagement based on stress coping styles is categorized according to the rise and fall patterns in hemodynamic parameters, as shown in Table 1 [23].

The symbol "+" in Table 1 indicates that the parameter has risen relative to baseline. Active engagement refers to active stress coping by increasing blood flow to skeletal muscle due to myocardial contractile activity and vasodilatation.
On the other hand, passive engagement is passive stress coping by contracting peripheral blood vessels. In addition, when MBP fell, it was classified as no stress coping. We identified ways of task engagement based on these stress coping styles. The measured hemodynamic parameters were normalized by subtracting the mean value during the Rest 2 period. The $8-13 \mathrm{~Hz}$ frequency component of the EEG is called the $\alpha$-wave and was used as an indicator of the central nervous system. The $\alpha$-wave is prominently expressed at rest, eye closure, and arousal, and decreases during eye opening and sleep. The normalized $\alpha$-wave power ratio was calculated by dividing the $\alpha$-wave power during the Rest 2 period. We focused on the nasal skin temperature (NST) as an index of sympathetic nervous system activity among the obtained FTIs [24]. Skin temperature is an autonomic nervous system index that depends on fluctuations in blood flow. Arteriovenous anastomoses (AVA), which regulate capillary blood flow, are concentrated around the nasal region. In addition, blood vessels in many parts of the face are located under the fat layer, whereas in the nasal area are located in a small gap between the nasal bone and the skin. Therefore, it is known that the physiological and psychological state of the body is markedly reflected in the nasal skin temperature [25]. Therefore, increased sympathetic nervous system activity decreases the blood flow, causing relative decreases in the NST. By contrast, inhibition of sympathetic nervous system activity increases the blood flow and causes a relative increase of NST [26]. Skin temperatures are affected by ambient conditions. By subtracting the skin temperature of the forehead from the NST, the ambient influences can be reduced [27]. For this study, the NST was adjusted by subtracting the mean of the right forehead skin temperature and the left forehead skin temperature. Following this, the

Table 1 Classification of stress coping styles

\begin{tabular}{llll}
\hline & MBP & CO & TPR \\
\hline Active stress coping & + & + & \pm \\
Passive stress coping & + & \pm & + \\
\hline
\end{tabular}

Fig. 1 Experimental protocol

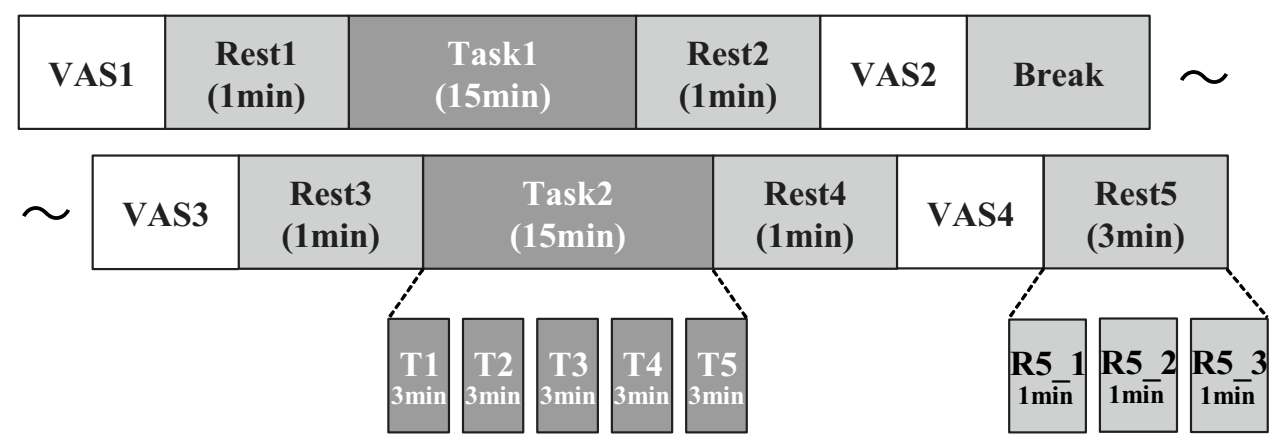


calculated NST was normalized by subtracting the mean value during the Rest 2 period.

The measured VAS scores of the subjective perception of feelings were used as psychological indices. For this study, the items evaluated were "Comfort", "Awareness", "Vigor" and "Refreshment" and they were rated by subjects lines using the adjective pairs "uncomfortable-comfortable", "sleepy-awake", "tired-sprightly" and "unrefreshing-refreshing", respectively. The differential values of VAS scores were calculated by subtracting the VAS scores of the "VAS2".

\section{Analysis methods}

\subsection{Evaluation segments}

Evaluation segments of the normalized hemodynamic parameters were defined as "T1", "T2", "T3", "T4" and "T5" by dividing the Task2 section into five 3-min sections. The mean in each segment was used as the evaluation value. For the evaluation segments of the $\alpha$-wave power ratio, we divided Rest 3 , Rest 4 , and Rest5 into three parts every minute (R5_1 to R5_3). The evaluation segments of the normalized NST were defined as Rest2, Rest3, T1 to T5, Rest4, R5_1 to R5_3. The mean in each segment was used as the evaluation value. The differential values of VAS scores were normalized by subtracting the VAS scores after Task1 (VAS2) from the VAS scores after the experiment (VAS4).

\subsection{Statistical evaluation}

For this study, statistical evaluation was conducted to compare the performance and psychophysiological states between the "Control" and "Intake" conditions. The Wilcoxon signed-rank test $\left(p<0.01^{* *}, p<0.05^{*}, p<0.1+\right.$ ) was used to compare the baseline for all indices, as well as the differential values of VAS scores and the rate of deterioration of TErr in the two conditions (Control and Intake). In addition, a two-way repeated analysis of variance (ANOVA) was used for all physiological indices to reveal whether the differences between the two conditions and the time lapse were statistically significant.

\section{Results and discussion}

\subsection{Performance evaluation}

Figure 2 shows the rate of deterioration of TErr, the evaluation value of the performance index. The horizontal axis indicates the conditions. The vertical axis indicates the evaluation values. The Wilcoxon signed-rank test found a significant difference between the baseline and mean values of the evaluation values in the Control condition $(p<0.01)$ as well as a significant difference between the two conditions $(p<0.05)$. This suggests that performance was maintained as a result of the food intake.

\subsection{Physiological evaluation}

Figure 3 shows the normalized hemodynamic parameters. The horizontal axis indicates the elements of each segment. The vertical axis indicates the normalized hemodynamic parameters. The Wilcoxon signed-rank test found a significant difference between the baseline and evaluation values in each condition $(p<0.01, p<0.05)$. The ANOVA found significant effects of the conditions $[F(1,23)=7.274$, $p<0.05]$ and time lapse $[F(4,92)=3.079, p<0.05]$ for MBP. Moreover, significant effects of time lapse $[F(4,92)$ $=38.805, p<0.01]$ and a significant interaction between the conditions and time lapse $[F(4,92)=6.838, p<0.01]$ were found for $\mathrm{CO}$. In addition, significant effects of time lapse $[F(4,92)=16.020, p<0.01]$ and a significant interaction between the conditions and time lapse $[F(4,92)=$ 6.803, $p<0.01]$ were found for TPR. The hemodynamic parameters show differences in subjects' stress coping styles between the two conditions. In the Control condition, the subjects have weak stress coping to the task. By contrast, in the Intake condition, subjects had a passive stress coping to the task in the beginning, but they had an active approach to it in the middle and later periods. In other words, the approach to the task is likely to be influenced by the food intake between tasks.

Figure 4 shows the $\alpha$-wave power ratio. The horizontal axis indicates the elements of each segment. The vertical axis indicates the $\alpha$-wave power ratio. An increase in the $\alpha$-wave power ratio means an increase in arousal. The Wilcoxon signed-rank test showed significant differences between the baseline and evaluation values in each condition as shown in Fig. $4(p<0.01, p<0.05)$. The ANOVA

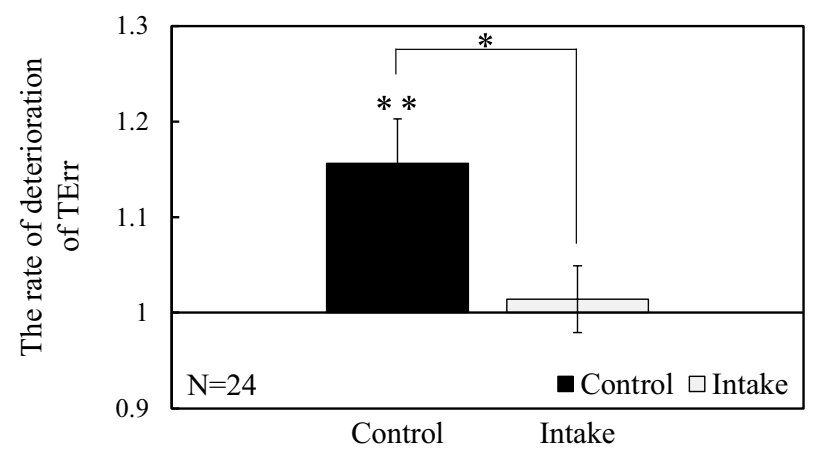

Fig. 2 The rate of deterioration of TErr $(N=24)$. The error bars represent the standard error $\left(p<0.01^{* *}, p<0.05^{*}, p<0.1+\right)$ 

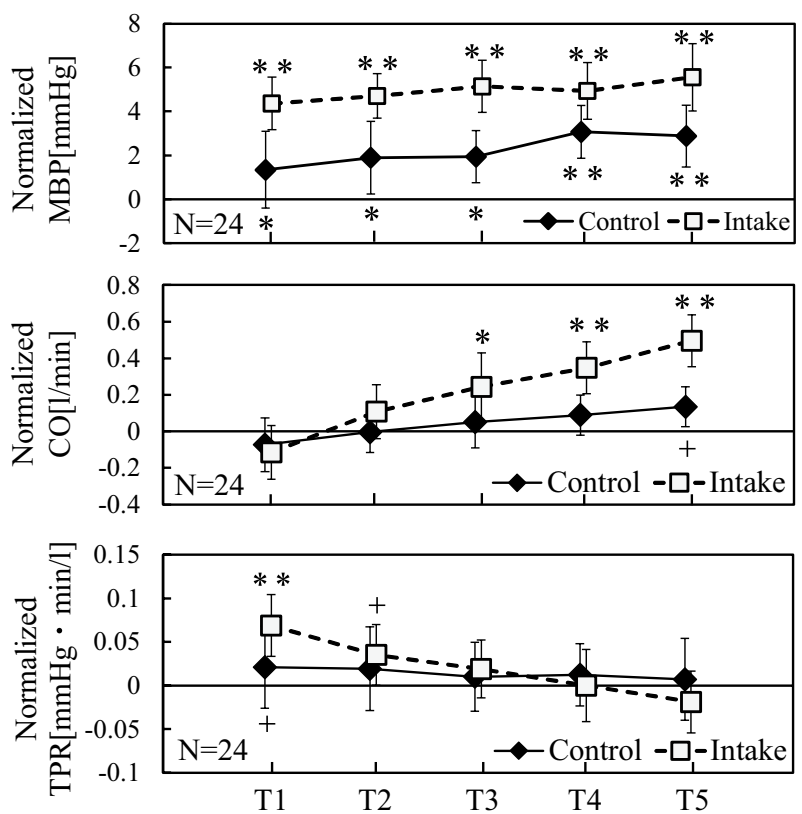

Fig. 3 Time-series variation of hemodynamic parameters $(N=24)$. The error bars represent the standard error $\left(p<0.01^{* *}, p<0.05^{*}\right.$, $p<0.1+)$

found significant effects of time lapse $[F(4,92)=4.476$, $p<0.01]$ were found.

Figure 5 shows the normalized NST. The horizontal axis indicates the elements of each segment. The vertical axis indicates the normalized NST. An increase in NST from baseline indicates a suppression of the sympathetic nervous system activity, while a decrease indicates an increasing sympathetic nervous system activity. The Wilcoxon signedrank test found significant differences between the baseline and evaluation values in each condition, as shown in Fig. 5 $(p<0.01, p<0.05)$. The ANOVA found significant effects of conditions $[F(1,23)=13.642, p<0.01]$ and time lapse $[F(10,230)=9.475, p<0.01]$, and a significant interaction

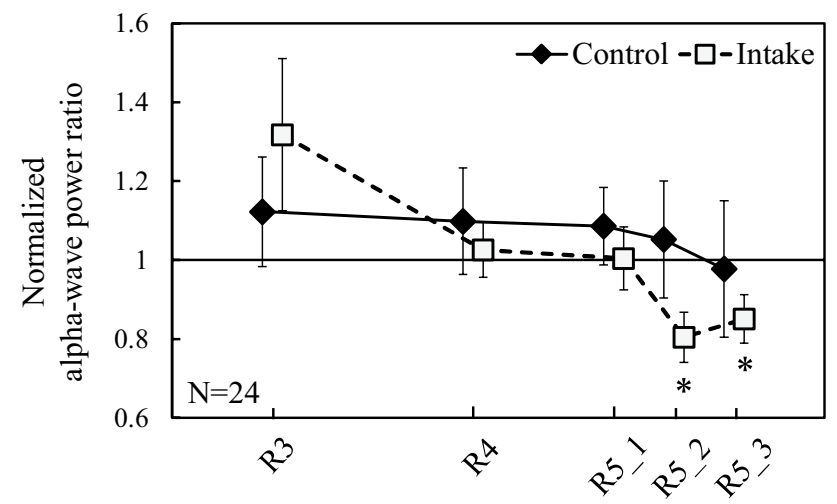

Fig. 4 The $\alpha$-wave power ratio in each segment $(N=24)$. The error bars represent the standard error $\left(p<0.01^{* *}, p<0.05^{*}, p<0.1+\right)$

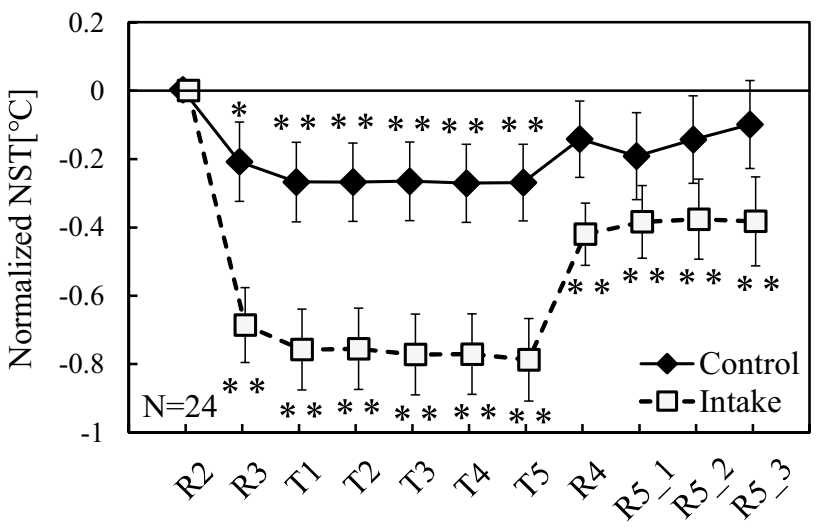

Fig. 5 The normalized NST in each segment $(N=24)$. The error bars represent the standard error $\left(p<0.01^{* *}, p<0.05^{*}, p<0.1+\right)$

between the conditions and time lapse $[F(10,230)=5.003$, $p<0.01]$.

In Intake condition, the $\alpha$-wave power ratio was significantly attenuated and the NST was significantly increased after the middle of Rest5. This may be due to the fatigue caused by the active engagement in the task. Furthermore, this fatigue was presumably appeared as a suppression of sympathetic nervous system activity and a sharp decrease in arousal level, triggered by closing eyes at rest.

\subsection{Psychological evaluation}

Figure 6 shows the differential values of the VAS scores. The horizontal axis indicates each assessment item of the subjective feelings. The vertical axis indicates the differential values. The Wilcoxon signed-rank test found significant differences in the baseline and evaluation values in each condition as shown in Fig. $6(p<0.01, p<0.05)$. The Wilcoxon signed-rank test found significant differences between the two conditions in all evaluation items of the subjective feelings $(p<0.01)$. All evaluation items of the subjective feelings were lower than baseline in the Control condition, but higher than baseline in the Intake condition. This suggests that psychological states throughout the task implementation were maintained by food intake. According to this result, it is thought that this is similar to the results indicated by the physiological indices, which confirmed the positive subjective feelings toward Task 2 in the Intake condition.

\section{Conclusions}

The objective of this study was to evaluate the effects of food intake on performance and task engagement based on psychophysiological states. The experiment was conducted under two conditions: resting with eyes open (Control) and 


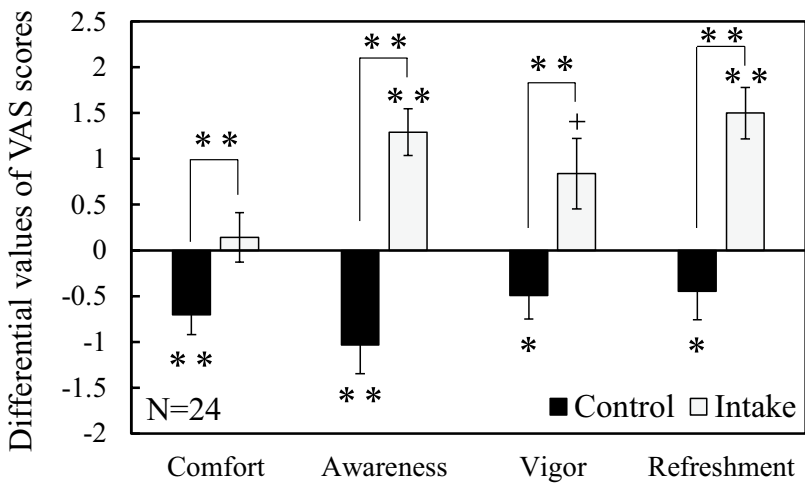

Fig. 6 The differential values of the VAS scores $(N=24)$. The error bars represent the standard error $\left(p<0.01^{* *}, p<0.05^{*}, p<0.1+\right)$

resting with food intake (Intake). We found that, in the Control condition, considering the responses of the cardiovascular system and NST during Task2, subjects did not actively engage in the task, and all assessment items of the subjective feelings were significantly lower than at the end of Task1. Therefore, task engagement was not improved by taking a break without eating food, and the performance of Task2 was worse than that of Task1. By contrast, in the Intake condition, sympathetic nervous system activity increased during Task2, and as a result of the change from passive to active engagement over time, the performance of Task 2 was maintained at the same level as that of Task1. Therefore, due to food intake between repeated tasks, psychological responses such as "Awareness" and "Refreshment" were maintained. Hence, it was confirmed that food intake stimulated positive task engagement in the task and maintained performance.

The experimental design of this study is limited in distinguishing between eating action and sensory factors such as flavor, texture, and nutrition. However, from the results, performance and task engagement based on the psychophysiological states were affected by eating action on performance and task engagement. Future work will mainly cover the what kinds of foods are actually suitable as stress-reducing, focusing on such as flavors and textures.

Open Access This article is licensed under a Creative Commons Attribution 4.0 International License, which permits use, sharing, adaptation, distribution and reproduction in any medium or format, as long as you give appropriate credit to the original author(s) and the source, provide a link to the Creative Commons licence, and indicate if changes were made. The images or other third party material in this article are included in the article's Creative Commons licence, unless indicated otherwise in a credit line to the material. If material is not included in the article's Creative Commons licence and your intended use is not permitted by statutory regulation or exceeds the permitted use, you will need to obtain permission directly from the copyright holder. To view a copy of this licence, visit http://creativecommons.org/licenses/by/4.0/.

\section{References}

1. World Health Organization, Mental health in the workplace, https://www.who.int/teams/mental-health-and-substance-use/ mental-health-in-the-workplace. Accessed 26 Oct 2021

2. Phyllis Gabriel, Marjo Riitta Liimatainen and International Labour Organization (2000), Mental health in the workplace, https://www.ilo.org/skills/pubs/WCMS_108221/lang--en/index. html, (accessed 2021-4-12)

3. Ministry of Health, Labour and Welfare, To-ward the realization of "Workplace Reform", https://www.mhlw.go.jp/stf/seisakunit suite/bunya/000 0148322.html, (accessed 2021-4-12)

4. Schaufeli WB, Taris TW, Rhenen W (2008) Workaholism, burnout and engagement: Three of a kind or three different kinds of employee well-being? Applied Psychology: an International Review 57(2):173-203

5. Bakker AB, Demerouti E (2008) Towards a model of work engagement. Career Development International 13(3):209-223

6. Schaufeli WB, Shimazu A, Taris TW (2009) Being driven to work excessively hard: The evaluation of a two-factor measure of workaholism in the Netherlands and Japan. Cross-Cultural Research 43(4):320-348

7. Schaufeli WB, SALANOVA M, GONZALEZ-ROMA V and Bakker AB, (2002) The measurement of engagement and burnout: A two sample confirmatory factor analytic approach. Journal of Happiness Studies 3:71-92

8. Kubota K, Shimazu A, Kawakami N (2014) Association of workaholism and work engagement with recovery experiences among Japanese workers. Japanese Journal of Behavioral Medicine 20(2):69-76 (in Japanese)

9. Ministry of Health, Labour and Welfare, The 1st year of Reiwa era "Analysis of the labor economy: Issues concerning work styles under the labor shortage", https://www.mhlw.go.jp/stf/wp/hakus yo/roudou/19/19-1.html. Accessed 26 Oct 2021

10. Sonnentag S, Mojza EJ, Demerouti E, Bakker AB (2012) Reciprocal relations between recovery and work engagement: The moderating role of job stressors. Journal of Applied Psychology 97(4):842-853

11. Sonnentag S, Binnewies C, Mojza EJ (2008) Did you have a nice evening? A day-Ievel study on recovery experiences, sleep, and affect, Journal of Applied Psychology 93:674-684

12. Sonnentag S, Mojza EJ, Binnewies C, Scholl A (2008) Being engaged at work and detached at home: A week-level study on work engagement, psychological detachment, and affect. Work \& Stress 22(3):257-276

13. Horie Y (1995) A study on optimum rest pause for VDT workers. Japan Industrial Management Association 46(3):225-231 in Japanese

14. Tanaka R, Sawai H, Koyama M (2015) Effects of Behaviors during Intermissions on Performance and Psychophysiological States. Comparison between the Text Conversation and the Oral Conversation, Time Studies 9:59-68 (in Japanese)

15. Yoneyama K, Komai H, Nishiguchi H, Okamura T, Yokomizo Y (1990) Influences of rest on VDT work, The Japanese Journal of. Ergonomics 26:190-191 (in Japanese)

16. Miki T, Terada T, Maeda T, Karasawa H, Adachi J, Tsukamoto M (2018) Investigating the Effect of How We Spend Our Break Time on Work Performance, The Special Interest Group Technical Reports of IPSJ, Vol. 2018-MBL-86. No. 3:1-8 (in Japanese)

17. Pagani M, Lombardi F, Guzzetti S, Sandrone G, Rimoldi O et al (1984) Power spectral density of heart rate variability as an index of symptho-vagal interactions in normal and hypertensive subjects. Journal of Hypertension Suppl 2(3):383-385 
18. Malliani A, Pagani M, Lombardi F, Cerutti S (1991) Cardiovascular neural regulation explored in the frequency domain. Circulation 84(2):482-492

19. Kurogi N, Nagumo K, Oiwa K, Nozawa A and Tominaga S (2019), Evaluation of time series feature of behaviour towards task, The 21th conference JSKS,13P-03, in Japanese

20. Kurogi N, Nagumo K, Oiwa K and Nozawa A (2020), Evaluation of time series characteristics of behaviour on task based on cardiovascular system and thermal system indices, The 19th Forum on Information Technology 2020, G-014, in Japanese

21. Sherwood A, Dolan CA, Light KC (1990) Hemodynamics of Blood Pressure Responses During Active and Passive Coping. Psychophysiology 27(6):656-668

22. Matsumura K, Sawada Y (2004) Controllability and hemodynamic reaction patterns during mental stress. Japanese Journal of Physiological Psychology and Psychophysiology 22(3):247-255 (in Japanese)

23. Lawler KA, Kline KA, Adlin RF, Wilcox ZC, Craig FW, Krishnamoorthy JS, Piferi RL (2001) Psychophysiological correlates of individual differences in patterns of hemodynamic reactivity. International Journal of Psychophysiology 40:93-107
24. Mizuno T, Nomura S, Nozawa A et al (2010) Evaluation of the effect of intermittent mental work-load by nasal skin temperature. IEICE Transactions on Information and Systems 93(4):535-543 (in Japanese)

25. Sawada Y (1998) Seirishinrigaku no Kiso: Taiontyousetukei) (Basics of Physiological Psychology: Body Temperature Regulation System. Kitaojisyobo, Kyoto, pp 222-235

26. Zenju H, Nozawa A, Tanaka H, Ide H (2004) Evaluation of unpleasant and pleasant states by nasal thermogram. IEEJ Transactions on Elevtronics, Information and Systems 124(1):213-214 (in Japanese)

27. Mizuno T, Nakategawa N, Kume Y (2012) Color influences on human beings evaluated by nasal skin temperature. Artificial Life and Robotics 16(4):519-522

Publisher's Note Springer Nature remains neutral with regard to jurisdictional claims in published maps and institutional affiliations. 